\title{
Novel Technique of Multislice CT Angiography for Diagnosis of Portosystemic Shunts in Sedated Dogs
}

\author{
Merja R. Laitinen', Jodi S. Matheson², Robert T. O’Brien² \\ ${ }^{1}$ Department of Equine and Small Animal Medicine, University of Helsinki, Helsinki, Finland \\ ${ }^{2}$ Department of Veterinary Clinical Medicine, University of Illinois, Urbana-Champaign, Urbana, USA \\ Email: merja.leinonen@helsinki.fi
}

Received February 1, 2013; revised March 7, 2013; accepted April 7, 2013

Copyright (C) 2013 Merja R. Laitinen et al. This is an open access article distributed under the Creative Commons Attribution License, which permits unrestricted use, distribution, and reproduction in any medium, provided the original work is properly cited.

\begin{abstract}
Diagnosing portosystemic shunts (PSS) can be challenging in veterinary patients. Multiple imaging techniques have been described to diagnose PSS. The aim of the study was to investigate whether a novel multi-slice computed tomography (CT) angiographic protocol could be used for diagnosis of PSS in dogs utilizing only patient sedation and without the need of test injection. Independent, blinded reviewers evaluated CT studies in a randomized order for study quality, shunt presence, number, and location of shunt origin and termination. Twenty two confirmed dogs were included in the study including 16 dogs with single extrahepatic congenital PSS, one dog with single intrahepatic congenital PSS, and two dogs with multiple acquired PSS. Three of the dogs (3/22) were surgically and histologically confirmed free of shunts. Final diagnosis was confirmed by surgery or necropsy. The new CT angiography protocol was shown to be diagnostic in dogs with PSS with no need for general anesthesia, test injections, delay times or timing determinations. Specificity and sensitivity were good $(100 \%)$. The quality of the studies was considered good in the majority of cases (20/22). The amount of motion artifact in the CT scans was minimal, and had no effect on the diagnostic quality. The CT protocol was found to be a useful, fast, and accurate tool for diagnosing portosystemic shunts with a 16-slice system.
\end{abstract}

Keywords: Dog; Portosystemic Shunt; Computed Tomography; Angiography

\section{Introduction}

Portosystemic shunts (PSS) are anomalous vessels arising from the portal vasculature and allowing the blood from the gastrointestinal tract and abdominal organs to bypass portal vasculature and empty into the systemic venous circulation [1-4]. Diagnosing PSS can be challenging in veterinary patients. Multiple imaging techniques have been described to diagnose PSS including mesenteric portography [4-10], splenoportography [10], per-rectal and trans-splenic portal scintigraphy (PRPS/ TSPS) [11-17], trans-splenic CT portography [18], CT and MRI angiography [19-25] and Doppler-ultrasonography [26-30]. CT angiography is considered the golden standards for imaging the portal vasculature in human medicine [31], however in veterinary medicine such a recommendation is missing, and both $\mathrm{CT}$ angiography and nuclear scintigraphy have been considered accurate enough. In general CT angiography is assumed to be the most accurate diagnosing method for PSS by many veterinarians, but this has not been scientifically tested ac- cording to the knowledge of the authors. In addition, all previously described CT protocols for diagnosing PSS involve pre-scan test injections for estimation of the best time to perform the scan [20,22-25]. Nuclear scintigraphy has been shown to have excellent sensitivity and specificity in diagnosing the presence or absence of PSS, however detailed anatomical information of the portal vasculature, including the origin and termination point of the shunt, cannot be assessed with PRPS [13-16]. In addition, radiation safety issues are a consideration with nuclear scintigraphy $[12,16,32]$.

The objective of the study was to evaluate a new CT protocol on dogs without general anesthesia. The hypothesis was that the amount of motion induced in sedated patients would not decrease the diagnostic quality of the scan and thus would be acceptable for clinical use. All previous CT protocols for the evaluation of PSS required general anesthesia [18,20,22-25]. Multi-slice CT has greatly reduced imaging time, whilst improving spatial resolution. With imaging times of multi-slice CT reduced to very short times (10 - 15 seconds per phase), 
the authors concluded it was possible to image small dog abdomen without general anesthesia. To the author's knowledge, no previous study has developed a protocol for the CT imaging of awake or sedated dogs for the diagnosis of PSS using multi-slice CT.

\section{Materials and Methods}

Twenty-nine dogs with suspected PSS were originally enrolled in the study, however seven of the dogs were excluded because no surgery or necropsy were performed to confirm the diagnosis. Finally twenty two dogs were included in the study. The inclusion criteria were the signalments, history, clinical signs and biochemical abnormalities were consistent with PSS. Before inclusion into the study each owner had to consent to CT angiography and exploratory celiotomy or necropsy. In dogs with no visible shunt, an exploratory laparatomy was performed to confirm the absence of the shunt, and hepatic biopsies were collected (3/22). The final diagnosis was confirmed surgery or necropsy in all patients (22/22). This protocol was approved by the Institutional Animal Care and Use Committee of the University of Illinois (09297).

Independent, blinded reviewers (J.M., R.O.B., and M.L.) evaluated CT studies in a randomized order for study quality and shunt presence, number and location of shunt origin and termination (anatomy). Study quality was assessed subjectively using following criteria: The study quality was considered non-diagnostic if the visualization of portal vasculature was obscured due poor spatial and contrast resolution and/or excess motion. The study quality was considered poor if portal vasculature and its major tributaries (cranial and caudal mesenteric veins, splenic vein and gastroduodenal vein) was poorly or only partially visible due poor spatial and contrast resolution and/ or motion obscuring the visualization of the shunt origin or termination. A good study quality was concluded when the portal vasculature with major tributaries and shunt anatomy was clearly visible, and additionally shunt origin and termination could be assessed. Final imaging diagnosis was reached by consensus. To blind the reviewers, 15 additional $\mathrm{CT}$ angiography cases, scanned outside the study with a slightly differing protocol, were included in the evaluation process. These additional cases were not included in the statistical analysis, except two cases that were diagnosed to have an extrahepatic portosystemic shunt during the consensus reading. Time between the CT angiograms and final evaluation was in minimum three months, to serve as blinding for the cases.

For the CT angiography, the majority of the dogs (19/22)

${ }^{a}$ Dexdomitor, Orion Pharma, Espoo, Finland.

${ }^{\mathrm{b}}$ Butorject, Phoenix Pharmaceutical, MO, USA.

${ }^{\mathrm{c}}$ Atropine Sulfate, Med-Pharmex, CA, USA.

${ }^{\mathrm{d}}$ Robinul-V, Fort Dodge Animal Health, IO, USA. were sedated with dexmedetomidin ${ }^{\mathrm{a}}$ (dose $0.0005-0.02$ $\mathrm{mg} / \mathrm{kg} \mathrm{IM})$, butorphanol ${ }^{\mathrm{b}}(0.2-0.4 \mathrm{mg} / \mathrm{kg} \mathrm{IM})$ added with atropine ${ }^{\mathrm{c}}(0.009-0.01 \mathrm{mg} / \mathrm{kg} \mathrm{IM})$ or glycopyrrolat $^{\mathrm{d}}$ (dose $0.01 \mathrm{mg} / \mathrm{kg} \mathrm{IM}$ ), and midazolam ${ }^{\mathrm{e}}$ (dose $0.2 \mathrm{mg} / \mathrm{kg}$ IV) when necessary. Three dogs were administered propofol $^{\mathrm{f}}(1-7 \mathrm{mg} / \mathrm{kg} \mathrm{IV})$ to induce sedation in cases where the previously mentioned sedation protocol was determined not appropriate due the clinical condition of the patient. However, no dogs were intubated or placed under general anesthesia during any CT angiography. No studies needed to be repeated for patient motion. Atipamezole ${ }^{\mathrm{g}}$ was used as same amount as single dose of dexmedetomidine to reverse sedation at the conclusion of all imaging procedures. During sedation the patients were monitored for pulse strength, heart rate and peripheral pulse.

Dogs were imaged in sternal recumbency, stabilized by external support (open cell foam blocks and wedges and CT system's velcro straps) on a multi-slice CT system ${ }^{\mathrm{h}}$. A lateral and dorsal scout was obtained on each dog and a single pre-contrast helical CT was obtained from the cranial-most aspect of the diaphragm to the level of the coxofemoral joints. Non-ionic contrast media $\left(660 \mathrm{mg} / \mathrm{kg}\right.$ of iodine, Ultravist ${ }^{\mathrm{i}}$ ) was manually injected as a fast bolus into an intravenous cephalic catheter. Imaging began immediately after the injection. Dogs were imaged using $250 \mathrm{~mA}$ and $120 \mathrm{kV}$, and a collimation/ slice thickness of $1.25 \mathrm{~mm}$, image reconstruction interval of $0.625 \mathrm{~mm}$, pitch of 1.3 , with speed of $13.75 \mathrm{~mm} / \mathrm{sec}$, and tube rotation time of $0.5 \mathrm{~s}$. Four consecutive postcontrast scans were obtained. The first three were the CT angiogram series and the dogs were imaged from the level of the dome of the diaphragm to the level of caudal pole of the left kidney. The last post-contrast scan extended caudally to the level of bladder neck region to evaluate for any other lesions (such as additional vascular abnormalities) in the caudal abdomen. Typical image time was 40 - 50 seconds for the 3 post-contrast CT angiography scans. In cases when the animal was very small (scan length $<200 \mathrm{~mm}$ ), with subsequent short imaging time, inter-scan delay was increased by $1-2 \mathrm{~s}$ and/or pitch was decreased to increase the time from the start of one phase to the start of the next phase to $10 \mathrm{~s}$ to insure the portal vascular phase was obtained. Thus the total time of the animal spent in the $\mathrm{CT}$ examination room was approximately 10 to 15 minutes. No postprocessed images (such as MPR or 3D images) were chosen to the subsequent evaluations and all images were reviewed using dedicated CT workstations ${ }^{j}$. The number

\footnotetext{
${ }^{\mathrm{e}}$ Midazolam, Bachem, Switzerland.

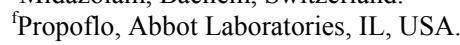

${ }^{\mathrm{g}}$ Antisedan, Orion Pharma, Espoo, Finland.

${ }^{\mathrm{h}} \mathrm{GE}$ lightspeed 16 slice CT, Wisconsin, USA.

${ }^{\mathrm{i} U}$ Ultravist, Bayer Healthcare Pharmaceuticals Inc., Germany.

${ }^{\mathrm{j}}$ Kodak, Health Imaging Division, NY, USA.
} 
of shunting vessels and the origin and termination of the shunt vessels were confirmed by visual estimation of the caval or portal phases on the original transverse-plane images. An arterial phase was included in the protocol, and it usually appeared during the first post-contrast scan. The quality of the arterial phase was, however not quantified as this was out of the scope of the study. The amount of motion in the CT scans was estimated visually by calculating the number of slices having motion artefact. The number, origin and termination of the PSS were determined as fully as possible, and the shunts were classified as single intrahepatic, single extrahepatic (Figure 1), or multiple extrahepatic. The single extrahepatic shunts were further subdivided into portocaval, splenocaval, portoazygos (Figure 1) and splenoazygos.

The proportion of motion noted in transverse images (per total amount of slices) in CT was calculated as [(number of slices where motion was detected divided by total number of slices) $\times 100$ ]. The number of margin incongruencies or "steps" in the sagittal reconstructed MPR image was reported as absolute values, one sagittal reconstruction per scan was performed. Both the proportion of motion in transverse slices (averaged by total amount of slices) and the number of steps in sagittal reconstruction images were calculated.

The sensitivity and specificity with $95 \%$ confidence interval in comparison to surgery or necropsy was calculated. Statistical significance was set at $p<0.05$. All statistical analyses were performed by 4Pharma Ltd using $\mathrm{SAS}^{\circledR}$ System for Windows, version $9.2^{\mathrm{k}}$. All statistical analyses were performed by 4 Pharma Ltd. using $\mathrm{SAS}^{\circledR}$ System for Windows, version $9.2^{\mathrm{k}}$.

\section{Results}

All of the dogs (22) included in the study were small or toy breeds $(1.1-8.5 \mathrm{~kg})$, with the exception of one medium sized dog $(29 \mathrm{~kg})$. All dogs confirmed to have a shunt had either increased pre-(14/14) and/or post-prandial (9/9) bile acids, and/or elevated blood ammonia levels $(8 / 8)$, unfortunately these were not measured consistently from all the patients as the major focus of this study was in imaging findings. PSS was diagnosed with CT angiography in 19/22 dogs; 16 dogs with single extrahepatic PSS ([8] portocaval, [4] splenocaval, [2] portoazygos, [2] splenoazygos), 1 dog with single intrahepatic PSS, and 2 dogs with multiple acquired PSS. Three of the dogs $(3 / 22)$ were surgically confirmed as not having a shunt and histopathological confirmed as microvascular dysplasia, all small breed dogs (one mixed, two maltes, size $2.5-3.2 \mathrm{~kg}$ ). No complications were recorded with the sedation protocols used, CT angiography or surgery.

\footnotetext{
${ }^{\mathrm{k}} \mathrm{SAS}$ Institute Inc., Cary, NC, USA.
}

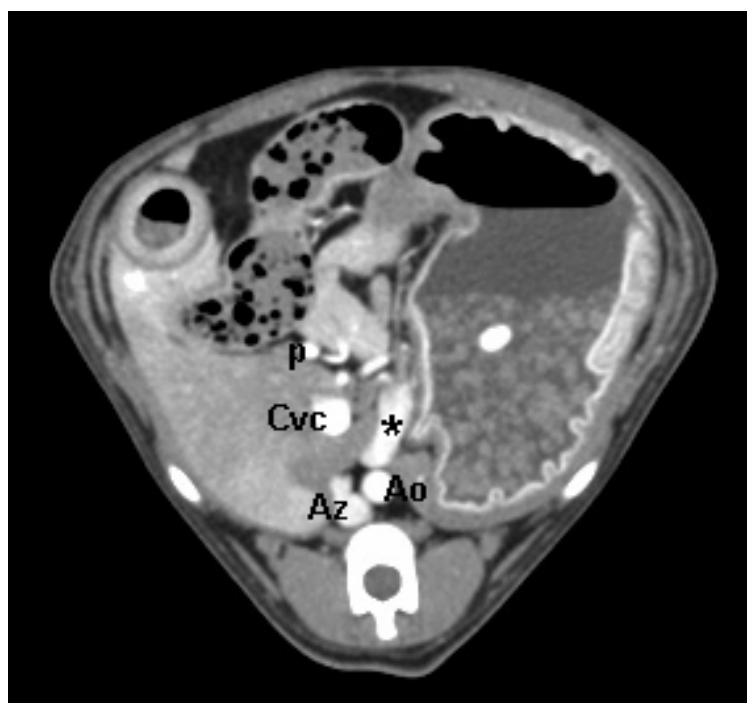

(a)

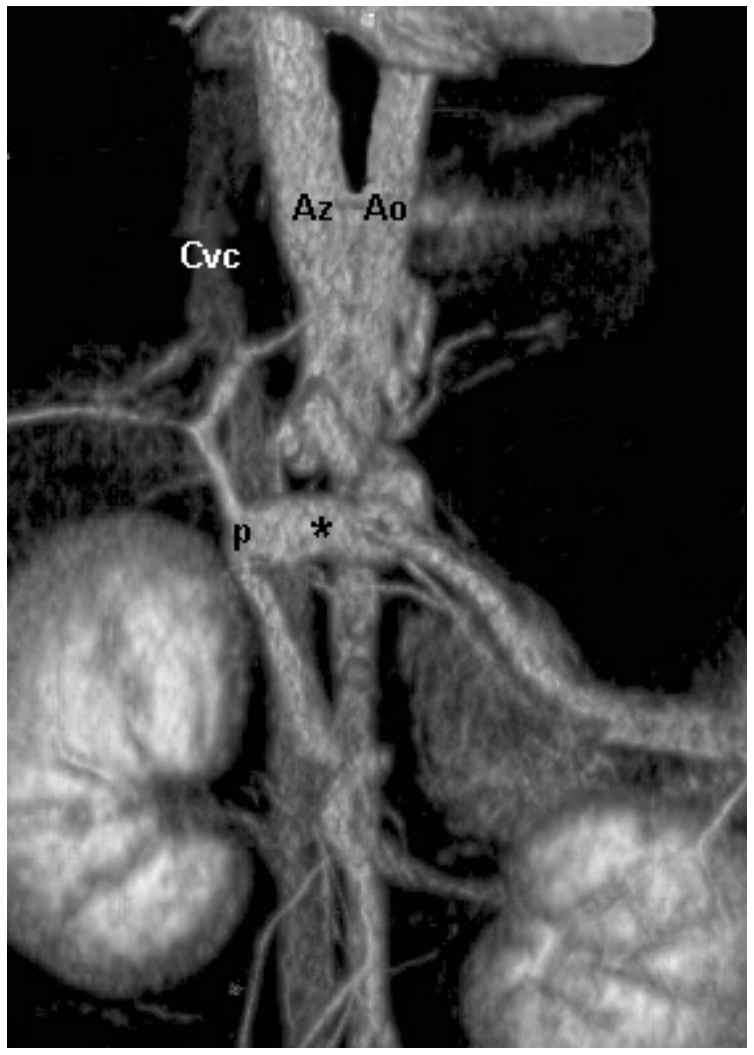

(b)

Figure 1. (a) Transverse CT image of an extrahepatic portoazygos shunt at the level of stomach, cranially to shunt origin from portal vein. An aberrant vessel is seen medially to the stomach and ventrally to aorta. Aorta (Ao), azygos vein (Az), shunt $(*)$, portal vein (p), caudal vena cava (Cvc); (b) CT 3D MIP reconstruction image of the same extrahepatic portoazygos shunt. An aberrant vessel is seen to travel from portal vein cranially, next to aorta, bypassing the liver and caudal vena cava. Aorta (Ao), azygos vein (Az), shunt $(*)$, portal vein (p), caudal vena cava (Cvc). Patient's head is in the top and right on the left of the image. 
The overall quality of CT angiographic studies was graded well in 20, and poor in 2 of the dogs. The reason for poor study quality was considered to be decreased detail due small patient size in one of the patients and the amount of step artifacts in the reconstructed images in one patient. However, the poor quality due step artifacts was not considered to disturb the diagnostic accuracy, rather complicate the estimation of exact shunt origin and/or termination. There were no non-diagnostic angiographies.

The amount of motion detected in the CT scans was subjectively estimated to be non-existent to mild in the majority of the patients $(21 / 22)$, and never affecting the diagnostic quality of the CT scans in such amount that it would compromise the diagnostic accuracy. Detected motion was low in the original transverse $(5.6 \%)$ and sagittal MPR images (2.4\%).

Specificity and sensitivity was good (100\%) for the used CT angiography protocol in comparison to surgery or necropsy (and histopathology). There were no false positive or false negative answers in the final evaluation, however two dogs had been falsely diagnosed free of shunts prior the study, that were later included in the study after surgical (single extrahepatic) or post-mortem confirmation (multiple extrahepatic) of the shunts. In majority of the cases the two first consecutive post-contrast scans were already diagnostic, leaving the third scan unnecessary for clinical use of the protocol.

The CT angiography protocol used in this study was accurate in detecting both the origin and termination point of the anomalous vessel in majority of the cases. However, there were two cases in which the termination of the shunts was missed in the original assessment by two of the readers (M.L., J.M.), likely due to small size of the vessel and atypically cranial termination of the shunt to caudal vena cava (near diaphragm, also termed as splenophrenic shunt [24]). Very thin collimation and short image reconstruction interval used in the study, were considered necessary in diagnosing these atypical and at times very small shunts in miniature sized dogs (smallest dog included in the study was $1.1 \mathrm{~kg}$ ).

After termination and evaluation of the study, new terminology has been proposed to use for more exact description of the shunt anatomy by Nelson and Nelson [24]. Such a detailed shunt anatomy would not have been possible in all of the cases, and more generalized terminology was thus used in this study. The reviewers felt that there were cases when it was not clear, whether the shunt originated exactly from the gastroduodenal or pancreaticoduodenal vein, or from left gastric or splenic vein, as the spatial resolution wasn't always good enough in very small patients. Furthermore, this level of detail was not considered necessary for the surgical treatment of the shunts in this study.

\section{Discussion}

In this study, multi-slice CT protocol with sedated patients was sensitive $(100 \%)$, specific $(100 \%)$, and accurate $(100 \%)$ method to diagnose PSS. Previous studies have reported excellent results for CT angiography in anesthetized patients, however no sensitivity has been evaluated/reported $[20,22,24,25]$. Our findings are consistent with previous studies [20,22], however the patients in this study were not anesthetized, but only lightly sedated (19/22) or administered low dose of iv-propofol $(3 / 22)$.

In this study, the diagnostic quality of the CT seemed not to be affected by lack of general anaesthesia. The hemodynamic effects of the sedation protocol used are likely to differ from general anesthesia, and this might have caused slightly different contrast enhancement compared to previous studies performed under general anesthesia. However, subjectively assessing and comparing the images with previous $\mathrm{CT}$ angiographies performed in the same institution this did not seem to have an effect on the contrast resolution of the scans. Three of the animals with seizures and signs of shock on physical exam were administered low dose propofol in lieu of dexmedetomidine, but these dogs were not intubated nor administered inhalant anesthesia. No post-procedural complications associated with sedation were observed in any patient.

The amount of motion artifact in the CT scans was detected to be minimal in all CT scans regardless of sedation protocol. Further studies are needed to compare the effects of sedation and general anesthesia on the contrast enhancement, spatial resolution and amount of detected motion.

CT protocols for sedated animals have not been previously published in veterinary patients suspected of having PSS. The multi-slice CT angiography protocol used in this study was easy to perform, as it required neither pre-scan test injection nor calculations of optimal timing for arterial and portal phases. Furthermore this technique was quick (40 - 50 seconds post-contrast image times), and no complications affecting to image quality (motion, other CT-artifacts) occurred during any of the CT procedures. The actual scan time in clinical use would be even shorter, considering that in maximum three post contrast scans will be required for accurate diagnosis, and to obtain both arterial and portal phases. Contrarily, in cases when the animal is very small leading to short imaging time, either an inter-scan delay must be increased or pitch decreased to increase the time from the start of one phase to the start of the next phase to insure the portal vascular phase will be obtained. This protocol is intended for multi-slice $\mathrm{CT}$ with isotropic pixel capabilities. The protocol is not suitable for single or 
dual slice CT systems.

The described CT protocol increased the amount of radiation exposure per patient $(341.53 \mathrm{mGy} / \mathrm{cm})$ in comparison to previously reported dual-phase CT protocol (approximately $200-300 \mathrm{mGy} / \mathrm{cm}$ depending on the size of the patient). However, even with this slight increase in the radiation exposure, the estimated radiation dose is considerably less than for example in a routine head or neck CT protocol. Furthermore, there were no non-diagnostic studies, and thus the small increase in radiation exposure was less than what can be expected in cases where the CT examination needs to be repeated due to bad timing of the test injection. In addition, in this study four consecutive post-contrast $\mathrm{CT}$ scans were performed to make sure that both arterial and portal vascular phases were included in the study. However, this will not be necessary in clinical use, as in majority of the cases the shunt vessel was visible already in the first two postcontrast scans. Further studies are needed to standardize this protocol and compare it with a dual-phase protocol. Additionally, radiation exposure could be decreased even further by excluding the pre-contrast scan from a shunt protocol as is currently done by some institutions.

\section{Conclusion}

In conclusion, CT angiography in sedated dogs was found to be a useful, fast, and accurate tool for diagnosing PSS using a 16-slice CT. Further comparisons with a conventional dual-phase CT protocol under general anesthesia are required for a detailed estimation of pros and cons in both protocols.

\section{Acknowledgements}

We thank the Dr. Allan L. and Mary L. Graham Endowment for financial support.

\section{REFERENCES}

[1] A. C. Berent and K. M. Tobias, "Portosystemic Vascular Anomalies," Veterinary Clinics of North America: Small Animal Practice, Vol. 39, No. 3, 2009, pp. 513-541. doi:10.1016/j.cvsm.2009.02.004

[2] D. R. Bostwick and D. C. Twedt, "Intrahepatic and Extrahepatic Portal Venous Anomalies in Dogs: 52 Cases (1982-1992)," Journal of the American Veterinary Medical Association, Vol. 206, No. 8, 1995, pp. 1181-1185.

[3] J. T. Payne, R. A. Martin and G. M. Constantinescu, "The Anatomy and Embryology of Portosystemic Shunts in Dogs and Cats," Seminars in Veterinary Medicine \& Surgery (Small Animal), Vol. 5, No. 2, 1990, pp. 76-82.

[4] J. Rothuizen, T. S. G. van den Ingh, G. Voorhout, et al., "Congenital Porto-Systemic Shunts in Sixteen Dogs and Three Cats," Journal of Small Animal Practice, Vol. 23, No. 2, 1982, pp. 67-81. doi:10.1111/j.1748-5827.1982.tb01638.x

[5] S. J. Bichard, D. S. Biller and S. E. Johnson, "Differentiation of Intra versus Extrahepatic Portosystemic Shunts in Dogs Using Positive-Contrast Portography", The Journal of the American Animal Hospital Association, Vol. 25, No. 1, 1989, pp. 13-17.

[6] N. J. Macdonald, C. A. Burton and R. N. White, "Comparison of Visual Analog and Numeric Scoring Scales for Assessing Intraoperative Mesenteric Portovenography," Veterinary Radiology \& Ultrasound, Vol. 43, No. 6, 2002, pp. 534-540. doi:10.1111/j.1740-8261.2002.tb01044.x

[7] S. Schmidt and P. F. Suter, "Angiography of the Hepatic and Portal Venous System in the Dog and Cat: An Investigative Method," Veterinary Radiology, Vol. 21, No. 2, 1980, pp. 57-77. doi:10.1111/j.1740-8261.1980.tb01353.x

[8] P. V. Scrivani, A. E. Yeager, N. L. Dykes and J. M. Scarlett, "Influence of Patient Positioning on Sensity of Mesenteric Portography for Detecting an Anomalous Portosystemic Blood Vessel in Dogs: 34 Cases (1997-2000)," Journal of the American Veterinary Medical Association, Vol. 219, No. 9, 2001, pp. 1251-1253. doi:10.2460/javma.2001.219.1251

[9] R. N. White, N. J. Macdonald and C. A. Burton, "Use of Intraoperative Mesenteric Portovenography in Congenital Portosystemic Shunt Surgery," Veterinary Radiology \& Ultrasound, Vol. 44, No. 5, 2003, pp. 514-521. doi:10.1111/j.1740-8261.2003.tb00499.x

[10] P. F. Suter, "Portal Vein Anomalies in the Dog: Their Angiographic Diagnosis," Veterinary Radiology, Vol. 16, No. 3, 1975, pp. 84-97. doi:10.1111/j.1740-8261.1975.tb01324.X

[11] G. Daniel, R. M. Bright, P. Ollis and R. Shull, "Per-Rectal Portal Scintigraphy Using ${ }^{99 \mathrm{~m}}$ Technetium Pertechnetate to Diagnose Portosystemic Shunts in Dogs and Cats," Journal of Veterinary Internal Medicine, Vol. 5, No. 1, 1991, pp. 23-27. doi:10.1111/j.1939-1676.1991.tb00926.X

[12] P. D. Koblik, J. Komtebedde, C. K. Yen and W. J. Hornof, "Use of Transcolonic ${ }^{99 \mathrm{~m}}$ Technetium-Pertecnetate as Screening Test for Portosystemic Shunts in Dogs," Journal of the American Veterinary Medical Association, Vol. 196, No. 6, 1990, pp. 925-930.

[13] P. D. Koblik, M. S. Hornof and W. J. Hornof, "Transcolonic Sodium Pertechnetate ${ }^{99 \mathrm{~m}} \mathrm{Tc}$ Scintigraphy for Diagnosis of Macrovascular Portosystemic Shunts in Dogs, Cats, and Potbellied Pigs: 176 Cases (1988-1992)," Journal of the American Veterinary Medical Association, Vol. 207, No. 6, 1995, pp. 729-733.

[14] F. Morandi, R. C. Cole, K. M. Tobias, et al., "Use of ${ }^{99} \mathrm{MCO}_{4}^{-}$Trans-Splenic Portal Scintigraphy for Diagnosis of Portosystemic Shunts in 28 Dogs," Veterinary Radiology \& Ultrasound, Vol. 46, No. 2, 2005, pp. 153-161. doi:10.1111/j.1740-8261.2005.00030.x

[15] F. Morandi, P. Sura, D. Sharp and G. B. Daniel, "Characterization of Multiple Acquired Portosystemic Shunts Using Transplenic Portal Scintigraphy," Veterinary Radiology \& Ultrasound, Vol. 51, No. 4, 2010, pp. 466-471. doi:10.1111/j.1740-8261.2010.01687.x 
[16] P. A. Sura, K. M. Tobias, F. Morandi, G. B. Daniel and R. L. Echandi, "Comparison of ${ }^{99 \mathrm{~m}} \mathrm{TcO}_{4}$ Trans-Splenic Portal Scintigraphy with Per-Rectal Portal Scintigraphy for Diagnosis of Portosystemic Shunts in Dogs," Veterinary Surgery, Vol. 36, No. 7, 2007, pp. 654-660. doi:10.1111/j.1532-950X.2007.00317.x

[17] B. J. van Vechten, J. Komtebedde and P. D. Koblik, "Use of Transcolonic Portal Scintigraphy to Monitor Blood Flow and Progressive Postoperative Attenuation of Partially Ligated Single Extrahepatic Porto-Systemic Shunts in Dogs," Journal of the American Veterinary Medical Association, Vol. 204, No. 11, 1994, pp. 1170-1174.

[18] R. L. Echandi, F. Morandi, W. T. Daniel, J. L. Paquette and G. B. Daniel, "Comparison of Transplenic Multidetector CT Portography to Multidetector CT-Angiography in Normal Dogs," Veterinary Radiology \& Ultrasound, Vol. 48, No. 1, 2007, pp. 38-44. doi:10.1111/j.1740-8261.2007.00202.x

[19] A. Bruehschwein, I. Foltin, K. Flatz, et al., "ContrastEnhanced Magnetic Resonance Angiography for Diagnosis of Portosystemic Shunts in 10 Dogs," Veterinary Radiology \& Ultrasound, Vol. 51, No. 2, 2010, pp. 116-121.

[20] P. Frank, M. Mahaffey, C. Egger and K. K. Cornell, "Helical Computed Tomographic Portography in Ten Normal Dogs and Ten Dogs with Portosystemic Shunt," Veterinary Radiology \& Ultrasound, Vol. 44, No. 4, 2003, pp. 392-400. doi:10.1111/j.1740-8261.2003.tb00475.x

[21] B. Seguin, K. M. Tobias, P. R. Gavin and R. L. Tucker, "Use of Magnetic Resonance Angiography for Diagnosis of Portosystemic Shunts in Dogs," Veterinary Radiology \& Ultrasound, Vol. 40, No. 3, 1999, pp. 251-258. doi:10.1111/j.1740-8261.1999.tb00357.x

[22] A. L. Zwingenberger, T. Schwarz and H. M. Saunders, "Helical Computed Tomographic Angiography of Canine Portosystemic Shunts," Veterinary Radiology \& Ultrasound, Vol. 46, No. 1, 2005, pp. 27-32. doi:10.1111/j.1740-8261.2005.00005.x

[23] A. L. Zwingenberger and F. S. Shofer, "Dynamic Computed Tomographic Quantitation of Hepatic Perfusion in Dogs with and without Portal Vascular Anomalies," American Journal of Veterinary Research, Vol. 68, No. 9, 2007, pp. 970-974. doi:10.2460/ajvr.68.9.970

[24] N. A. Nelson and L. L. Nelson, "Anatomy of Extrahepatic

\section{Abbreviations}

Computed tomography $=\mathrm{CT}$

Magnetic resonance imaging $=$ MRI
Portosystemic Shunts in Dogs as Determined by Computed Tomography Angiography," Veterinary Radiology \& Ultrasound, Vol. 52, No. 5, 2011, pp. 498-506. doi:10.1111/j.1740-8261.2011.01827.x

[25] G. Bertolini, E. C. Rolla, A. Zotti and M. Caldin, "ThreeDimensional Multislice Helical Computed Tomography Techniques for Canine Extra-Hepatic Portosystemic Shunt Assessment," Veterinary Radiology \& Ultrasound, Vol. 47, No. 5, 2006, pp. 439-443. doi:10.1111/j.1740-8261.2006.00173.x

[26] M. A. D’Anjou, D. Pennick, L. Cornejo and P. Pibarot, "Ultrasonographic Diagnosis of Portosystemic Shunting in Dogs and Cats," Veterinary Radiology \& Ultrasound, Vol. 45, No. 5, 2004, pp. 424-437. doi:10.1111/j.1740-8261.2004.04076.x

[27] D. E. Holt, C. G. Schelling, H. M. Saunders and R. J. Orsher, "Correlation of Ultrasonographic Findings with Surgical, Portographic, and Necropsy Findings in Dogs and Cats with Portosystemic Shunts: 63 Cases (19871993)," Journal of the American Veterinary Medical Association, Vol. 207, No. 9, 1995, pp. 1190-1193.

[28] C. R. Lamb and R. N. White, "Morphology of Congenital Intrahepatic Portocaval Shunts in Dogs and Cats," Veterinary Record, Vol. 142, No. 3, 1998, pp. 55-60. doi:10.1136/vr.142.3.55

[29] V. Szatmari, J. Rothuizen, T. S. G. van den Ingh, et al., "Ultrasonographic Findings in Dogs with Hyperammonemia: 90 Cases (2000-2002)," Journal of the American Veterinary Medical Association, Vol. 224, No. 5, 2004, pp. 717-727. doi:10.2460/javma.2004.224.717

[30] R. H. Wrigley, J. L. Konde, P. D. Park and J. L. Lebel, "Ultrasonographic Diagnosis of Portocaval Shunts in Young Dogs," Journal of the American Veterinary Medical Association, Vol. 191, No. 4, 1987, pp. 421-424.

[31] K. P. Henseler, M. A. Pozniak, F. T. Lee and T. C. III Winter, "Three-Dimensional CT Angiography of Spontaneous Portosystemic Shunts," Radiographics, Vol. 21, No. 3, 2001, pp. 691-704.

[32] R. C. Cole, F. Morandi, J. Avenell and G. B. Daniel, "Trans-Splenic Portal Scintigraphy in Normal Dogs," Veterinary Radiology \& Ultrasound, Vol. 46, No. 2, 2005, pp. 146-152. doi:10.1111/j.1740-8261.2005.00029.x

Per-rectal portal scintigraphy $=$ PRPS

Portosystemic shunt $=$ PSS

Trans-splenic portal scintigraphy $=$ TSPS 